\title{
Recursion applied to the Erlang $B$ formula for determining the telephone exchanges Grade of Service
}

\author{
Elieser E. Gallego Martínez¹, Jany Alpuín Martínez² \\ 1 Department of Telecommunications and Electronics, University of Pinar del Río Hermanos \\ Saíz Montes de Oca. \\ 2 Oficina Privincial del Ministerio de Comunicaciones en Pinar del Río. \\ elieserernesto@gmail.com, jany.alpuinm@gmail.com
}

\begin{abstract}
This paper describes how it is possible to apply a recursion to the original formula of Erlang B., to solve the problem of evaluating large numbers in that formula, using mathematical assistants, in applications designed to determine the grade of service in telephone exchanges.
\end{abstract}

Keywords. Erlang B., Grade of Service, GoS, Matlab.

\section{Recursividad aplicada a la fórmula de Erlang B para la determinación del Grado de Servicio en Centrales Telefónicas.}

Resumen. Este artículo describe cómo es posible aplicar una recursión a la fórmula original de Erlang B., para resolver el problema de evaluar números grandes en esa fórmula, usando asistentes matemáticos, en aplicaciones diseñadas para determinar el grado de servicio en las centrales telefónicas.

Palabras clave. Erlang B., Grado de Servicio, GoS, Matlab.

\section{Introduction}

Erlang $B$ formula is one of the most significant applications of queuing theory to the analysis of telecommunications traffic.

Specifically, this formula enables to determine the probability that a telephone service's subscriber cannot be handled when trying to make a call, or the likelihood that all facilities in a service system are occupied simultaneously, which obviously would be another reason why fails another new call attempt. This probability is called Grade of Service (It is defined by Equation (1) known as Erlang formula, or Grade of Service GoS) is denoted by the letter B.

$$
B=\frac{\frac{A_{0}^{N}}{N !}}{\sum_{i=0}^{N} \frac{A_{0}^{i}}{i !}}
$$

The terms $A o$ and $\mathrm{N}$ represent the intensity of offered traffic and the number of facilities respectively. The term facilities is related, in telephone systems, with the trunk circuits. See Figure 1.

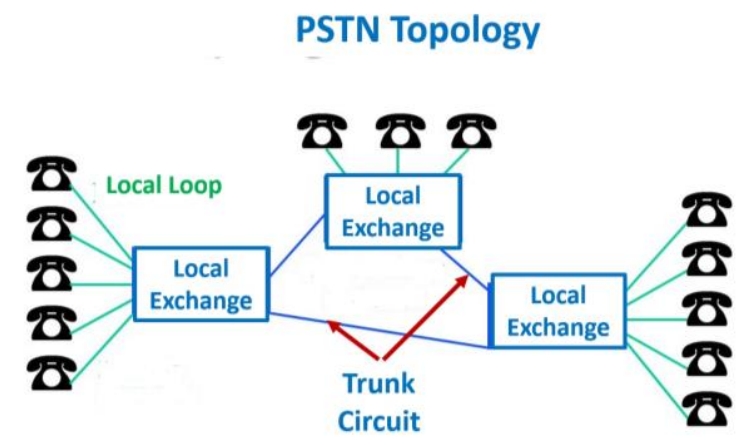

Fig. 1. Trunk circuits in the structure of a telephone network.

The instantaneous traffic intensity in a telecommunications system, when it is comprised of a set of resources (such as servers, circuits, lines, trunks, channels, etc.), is the number of resources occupied in a given moment [1]. The unit assigned to voice traffic is Erlang (Erl), in honor to the Danish Agner Krarup Erlang, who made important contributions to the theory of queues. The expression of Equation (2) is usually used to determine the intensity of traffic offered to a telephone exchange. 


$$
A_{o}=\frac{C * t_{p r}}{T_{o b}}
$$

Where $C$ represents the number of call attempts, $t_{p r}$ the average retention time of those calls, and $T_{o b}$ the System observation time. When the measurement unit selected to express traffic is the Erl, then this parameter, $T_{o b}$ takes the value of 1 hour, and this hour must be the busy hour.

Equation (1) it is widely used because it allows service providers to know precisely the amount of resources in which to invest, to provide a given level of service, knowing the demand of its customers in terms of offered traffic.

There are several conditions to apply this formula:

> It should be applied in systems operating under a service criteria of missed calls. That is, the behavior of the system to a new attempt to call, when all the center facilities are busy, is to reject with busy tone.

> The system must have full accessibility. That is, all entries must have access to all outputs.

$>$ The arrival time of call should respond to a negative exponential probability distribution.

$>$ The number of sources must be much greater than the number of facilities and independent among them.

For the work of engineers, this parameter is expressed in terms of 1 in many. For example, for a system in which every twenty calls is likely that at least one is blocked, the GoS of the system should be $1 / 20$, or what is the same 0.05 as the probability of not being able to set up the call on the first try .

The sector Telecommunication Standardization of the ITU (International Telecommunication Union) recommends designing the national network links with a probability of loss not greater than $1 \%$ [2], which means that commercially a GoS of $1 / 100$ is acceptable.

Although the worst degree of global service corresponds approximately to the sum of the loss probabilities of different links connected in cascade, in most global communications service level will be significantly better.

\section{Problem Statement}

It is a trend of service providers to take a rigorous call completion record and the extent of how this affects the Grade of Service.

In digital telephone exchanges, the completion of calls is commonly tabulated in data files containing a widely detailed statistics of events related to telephone calls. Note in the following figure the behavior of how traffic intensity tends to shoot for an hour a day.

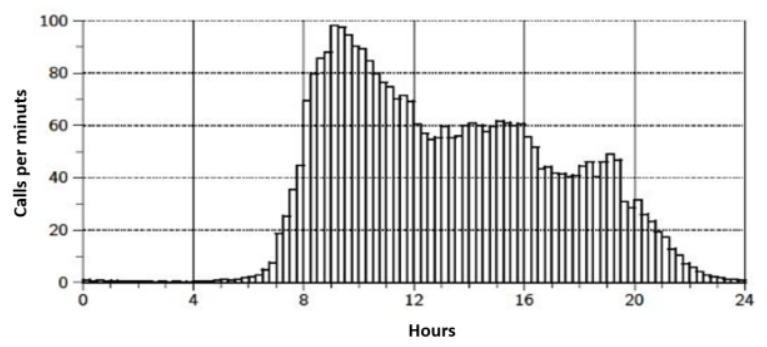

Fig. 2. Behavior of the average calls on a telephone exchange.

That time is called busy hour, and all statistical analyzes traffic in telephone systems are relative to that time. In the above figure it corresponds to the time that elapses between 9 and $10 \mathrm{am}$. For example, to determine the GoS in telephone exchanges of small or medium complexity and size, knowing the number of trunks, having a traffic offered average during the busy hour, to perform the calculation with MATLAB, evaluating Equation (1) of the previous section, these values would be the result shown in Figure 3. 




Fig. 3. GoS behavior for small and medium size telephone exchanges.

As seen in Figure 3, for telephone exchanges whose dimensions exceed 150 trunks, when the traffic intensity approaches that amount, it is not possible to determine the GoS, even using powerful mathematical assistants as MATLAB, whose maximum positive real number is $1.7977 \mathrm{e}+308$, in the MATLAB R2014a version.

In the particular case of the above example, when the offered traffic was 130 and 150 Erl., the obtained results for the GoS were the values shown on Table 1, corresponding to the last ten trunks, number 121 to the 131:

$\mathrm{NaN}$ returns the IEEE $\AA$ arithmetic representation for Not-a-Number $(\mathrm{NaN})$. These values result from operations which have undefined numerical results [3].

It is needed to say that, although the value of the GoS is small in terms of probabilities, it can be interpreted as significant economic losses for service providers, and a considered numbers of unsatisfied clients. But, for big dimension centrals, as those installed on the borders of the countries, set to meet international traffic, the situation is even more critical, because in periods called peak, the traffic intensity shoots up very close to values to the number of resources available.

There are some aspects to emphasize in this situation:

The average use of the facilities is bigger for big groups.

$>\quad$ If the offered traffic is increased, then the probability of lost a call increases for all value of $\mathrm{N}$. Therefore, it is necessary to decide possible options between two:

1. A high sensibility to traffic overload.

2. A low use of the trunks [4].

These two aspects can be seen in the Figure 4.

Everything exposed here, shows the need to increase the accuracy in determining the grade of service in telephone exchanges. 
Table 1. GoS values for the last ten trunks of the example of Figure 3.

\begin{tabular}{cccccccccccc}
\hline Offered & \multicolumn{10}{c}{ GoS / Number of Trunks } \\
Traffic (Erl.) & 140 & 141 & 142 & 143 & 144 & 145 & 146 & 147 & 148 & 149 & 150 \\
\cline { 2 - 12 } 130 & 0.0282 & 0.0253 & 0.0226 & 0.0202 & 0.0179 & 0.0158 & NAN & NAN & NAN & NAN & NAN \\
150 & 0.1086 & 0.1036 & NAN & NAN & NAN & NAN & NAN & NAN & NAN & NAN & NAN \\
\hline
\end{tabular}

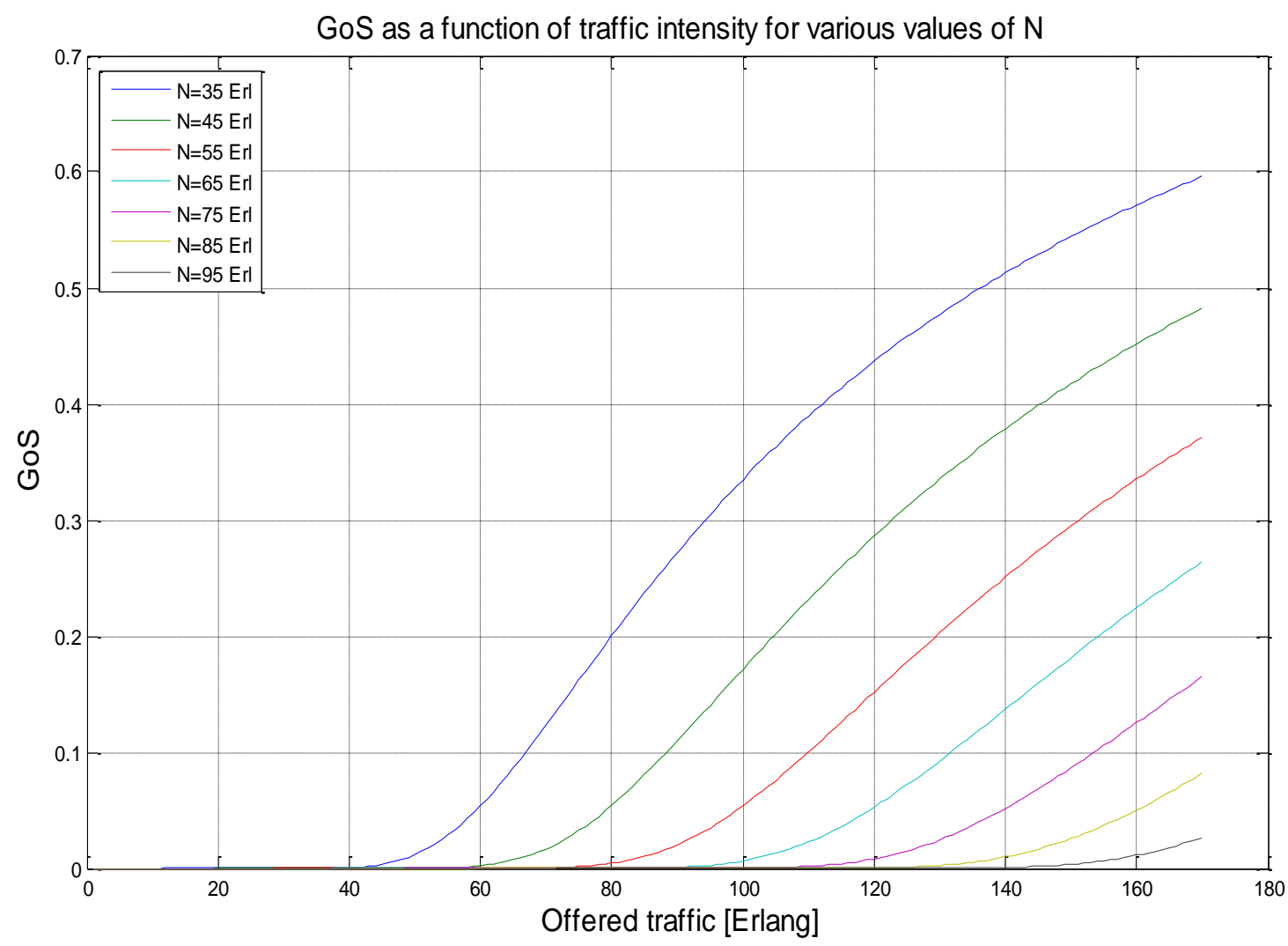

Fig. 4: GoS as a function of the offered traffic intensity for various values of $\mathrm{N}$. 


\section{Discussion and Results}

in this section we show how to use recursive technique to get a numerical fine solution to the Erlang $\mathrm{B}$ formula.

Starting from Erlang B formula in the Equation (1), and evaluating $n=1$ :

$$
\mathrm{B}=\frac{\mathrm{A}}{1+\mathrm{A}}=\frac{1}{1+\frac{1}{\mathrm{~A}}}
$$

Making:

$$
\mathrm{r}_{1}=1+\frac{1}{\mathrm{~A}}
$$

Then, if $n=2$ :

$$
B=\frac{A^{2}}{2\left(1+A+\frac{A^{2}}{2}\right)}=\frac{1}{\frac{2}{A^{2}}+\frac{2}{A}+1}=\frac{1}{1+\frac{2}{A}\left(1+\frac{1}{A}\right)}
$$

Substituting $r 1$ in (5):

$$
B=\frac{1}{1+\frac{2}{A} * r_{1}}
$$

Making:

$$
r_{2}=1+\frac{2}{A} * r_{1}
$$

If $\mathrm{n}=3$ :

$$
B=\frac{A^{3}}{6 *\left(1+A+\frac{A^{2}}{2}+\frac{A^{3}}{3 !}\right)}=\frac{1}{1+\frac{3}{A}+\frac{6}{A^{2}}+\frac{6}{A^{3}}}=\frac{1}{1+\frac{3}{A} *\left[1+\frac{2}{A} *\left(1+\frac{1}{A}\right)\right]}
$$

Substituting r2 in Equation (8)

$$
B=\frac{1}{1+\frac{3}{A} * r_{2}}
$$

Generalizing the result:

And finally:

$$
r_{n}=1+\frac{n}{A} * r_{n-1}
$$

$$
B=\frac{1}{1+\frac{n}{A} * r_{n-1}}
$$

This result allows sequential programming of Erlang original formula without losing the accuracy of the final result, while ensuring that at any time we work with large numbers, which may truncate processing.

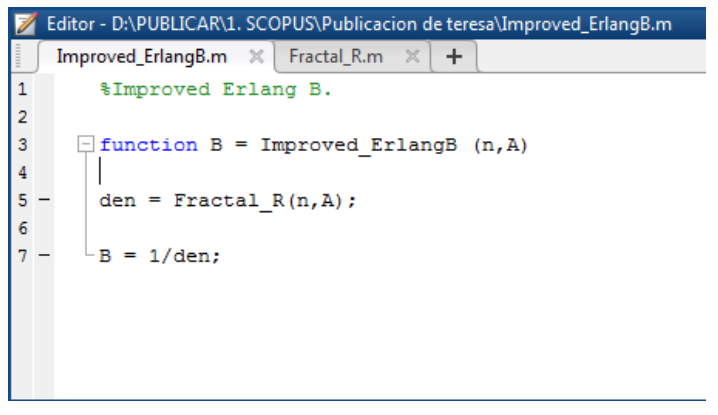

Fig. 5. Source code of the Equation (11) in a Matlab implementation.

In general, the proposal from programming would be the following algorithm for the implementation of the Erlang formula $B$ (Figure 5):

And the invoked "Fractal_R.m" function is in the Figure 6.

The next table shows the obtained results of the evaluation of the example shown in the Table 1, with the new methodology defined in the functions shown in Figures (5) and (6):

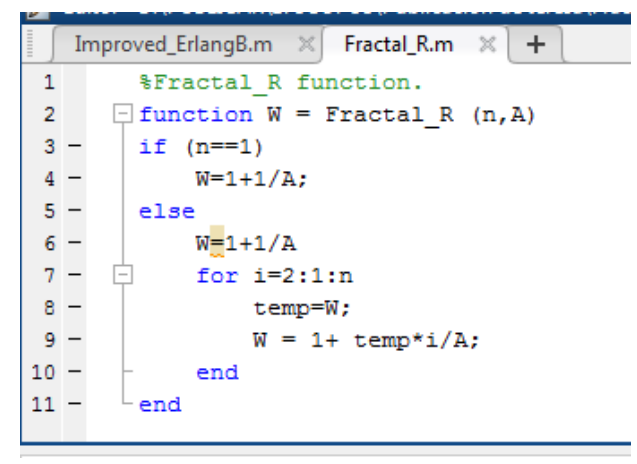

Fig. 6. Source code of the the Equation (10) in a Matlab implementation. 
Table 2. GoS values for tha last ten trunks of the example of Figure 3 using the new .

\begin{tabular}{|c|c|c|c|c|c|c|c|c|c|c|c|}
\hline \multirow{2}{*}{$\begin{array}{c}\text { Offered } \\
\text { Traffic (Erl.) }\end{array}$} & \multicolumn{11}{|c|}{ GoS / Number of Trunks } \\
\hline & 140 & 141 & 142 & 143 & 144 & 145 & 146 & 147 & 148 & 149 & 150 \\
\hline 130 & 0.0282 & 0.0253 & 0.0226 & 0.0202 & 0.0179 & 0.0158 & 0.0139 & 0.0121 & 0.0105 & 0.0091 & 0.0078 \\
\hline 150 & 0.1086 & 0.1036 & 0.0986 & 0.0938 & 0.0890 & 0.0843 & 0.0797 & 0.0752 & 0.0708 & 0.0666 & 00624 \\
\hline
\end{tabular}

The point here is that there are not undefined numerical results as in the case of Table 1. Those results are remarked in red.

\section{Conclusions}

It has been demonstrated that recursion is a tool that facilitates performing mathematical operations that require a high computational cost.

In terms of probability of being not able to establish a call during the busy hour, the proposed methodology provides a parameter that accurately measures the performance of a complete set of calls, which allows a more consistent planning with the real needs of service providers and its customers.

\section{References}

[1] Rincón Rivera, D. (1998) Introducción a los modelos de tráfico para redes de banda ancha.

[2] Unión Internacional de las Telecomunicaciones UIT-T Sector de Normalización de las Telecomunicaciones de la UIT, E.541. (1993). Grado de Servicio Global en las Conexiones Internacionales.

[3] http://www.mathworks.com/help/matlab/r ef/nan.html

[4] Study Group 2 ITU-D. (2006). Teletraffic Engineering. 